\title{
Contribución al estudio de las blastomicosis en Costa Rica
}

\author{
por \\ A. Trejos ${ }^{* *}$ y Arturo Romero*** \\ (Recibido para su publicación el 14 de mayo de 1953)
}

De las Granulomatosis Blastomicoides o Blastomicosis, han sido señalados en Costa Rica únicamente tres casos: dos de enfermedad de Lutz (10-11-13) y uno en el cual se aisló Torulopsis mitis (actualmente Rbodotorula mitis) (9).

Los anteriormente publicados como "Blastomicosis tipo Gilchrist" (7-8-12) no tuvieron confirmación micológica, único medio de escablecer el diagnóstico específico. Por esta razón, así como por la sintomatología presentada por los enfermos, y tomando en cuenta la distribución geográfica de la Blastomicosis Norteamericana, creemos se tratara de otras enfermedades que fueron confundidas con la de Gilchrist.

Hemos tenido oportunidad de encontrar y confirmar un nuevo caso de enfermedad de Lutz y el primer caso costarricense de Blatomirosis Queloideana o Enfermedad de Lobo, siendo éste el primero señalado fuera de la región Amazónica, y el octavo en el mundo, según los últimos datos de Lово (6). ${ }^{3}$

* Trabajo presentado al $5^{\circ}$ Congreso Internacional de Microbiolıgía efectuado en Río de Janeiro del 17 al 24 de agosto de 1950.

* Laboratorio Bacteriológico del Hospital San Juan de Dios.

* Servicio de Enfermedades Infecto Contagiosas del Hospital San Juan de Dios (Dirección actual).

1 En agosto de 1950 publican Guimaraes y Macedo (Guimaraes, F. N. \& Macedo, D. G., 1950. Contribuicao ao estudo das blastomicoses na Amazonia. O Hospital 38 (2) : 223-253) una revisión de los casos de blastomicosis oueloideana y la descripción de uno nuevo. Incluyendo el nuestro, a que hacen referencia los antes mencionados autores, habría un total de nueve conocidos. Algunos consideran èl caso de da Fonseca producido por Glenosporopsis amazonica como siendo la misma entidad clínica. Este punto de vista elevaría el número total de casos a diez. 
Los estudios detallados de estos enfermos serán publicados posteriormente y aquí nos limitaremos a resumirlos de la manera siguiente:

\section{GRANULOMATOSIS BLASTOMICOIDE NEOTROPICAL}

\section{O ENFERMEDAD DE LUTZ}

R. Z. de 61 años, agricultor, vecino de Platanillo de Turrialba (región Este del país sobre la vertiente del Atlántico) ingresa al Hospital el 19 de diciembre de 1948.

Hace tres meses presentó diarrea y cansancio. Después, un nódulo submentoniano de aumento progresivo. Posteriormente apareció una pápula en el surco nasogeniano. Sólo entonces notó que una prótesis total inferior pro. vocaba dolor en el reborde dentario (Plancha I, Fig. 1). Aun cuando el enfermo refiere como primer síntoma la diarrea, creemos que la lesión primaria fué la de la encía, al nivel de los incisivos inferiores derechos.

Cuando ingresó al Hospital presentaba una úlcera de fondo frambuesiforme de unos $2 \mathrm{~cm}$ de diámetro en el surco nasogeniano; otra de las mismas dimensiones en la planta del pie derecho; pápulas en la frente y ambas mejillas, una turnoración fluctuante en la región submaxilar izquierda, y una lesión vegetante epiteliomatosiforme de la encía. Ganglios axilares y del cuello aumentados de volumen. Meteorismo acentuado, diarrea con promedio de ocho deposiciones diarias. Tos ligera. El enfermo falleció sin que la terapéutici: a base de sulfamidados y estroptomicina surtiera ningún efecto. Debido a incomprensión de los parientes del mismo, no fué posible practicarle autopsia completa, habiendo tenido que limitarnos a extraer el intestino a través de una pequeña incisión. Este mostró numerosas ulceraciones tres de las cuales estaban perforadas.

Según la clasificación de Aguiar Pupo (1) a que hace referencia AlmeiDA (2) este cuadro debe ser clasificado como una "Forma mixt?" por presentar en la fase final de su evolución multiplicidad y polimorfismo de lesiones.

El examen microscópico a fresco, en extensiones coloreadas, con Giemsa y en cortes de material retirado por biopsia y autopsia, demostró la presencia de Paracoccidioides brasiliensis en todas las lesiones cutáneas $g$ de la encía, sea libres, sea en el interior de histiocitos y gigantocitos (PI. I, Figs. 3-5-6-7), así como también en las heces, el esputo y los cortes de intestino (PI. I, Fig. 4).

El estudio del agente etiológico se completó con el cultivo obtenido del pus extraído por punción del ganglio submaxilar (Pl. I, Fig. 2), así como también por la inoculación intratesticular del mismo material en cobayos que desarrollaron una orquitis supurada de la cual pudo obtenerse otro cultivo.

En lo que a morfología del $P$. brasiliensis se refiere, pudimos observar, en el material obtenido por raspado de las lesiones cutáneas, sea coloreado con Giemsa o a fresco, formas típicas de criptosporulación (Pl. I, Fig. 3.-Pl. II, Fig. 3). 
En las preparaciones coloreadas por Giemsa las criptosporas se presentan aparentemente desprovistas de citoplasma y coloreadas en rojo brillante intenso, rodeadas de una fina membrana que aparece como un halo claro. Estas criptosporas miden 0,7 a 1,5 micras y las encontramos en gran cantidaci en algunas de las lesiones de la mucosa intestinal (Pl. I, Fig. 4).

En material de encía retirado por biopsia se encontró también una célula en criptosporulación que muestra perfectamente los agujeros de la membrana de más o menos 0,4 micras, a través de los cuales salen la criptosporas (Pl. II, Figs. 1 y 2).

Además de los métodos de esporulación por todos conocidos, se ha señalado la posibilidad de que el $P$. brasiliensis presente endosporulación; po: esta razón dedicamos especial cuidado al estudio de una célula en la que tal proceso podía sospecharse (Pl. II, Figs. 4 y 5). Pudimos comprobar que las estructuras internas que se observan en estas células son de naturaleza lipoide, coloreándose electivamente con el Sudan III. Además estas vacuolas son particularmente abundantes cuando las células, conservadas en gota pendiente, emiten tubos germinativos (Pl. II, Figs. 6 y 7), por lo que se puede presumir que tienen su metabolismo acelerado. Siguiendo este razonamiento y tomando en consideración que los anticuerpos que se producen en este tipo de micosis son principalmente aquellos fijadores del complemento, cabe formular la hipótesis de que la toxina del $P$. brasiliensis sea una substancia liposoluble o de naturaleza lipoide, y efectuar las experiencias del caso para dilucidar este punto.

\section{BLASTOMICOSIS QUELOIDEANA O ENFERMEDAD DE LOBO}

A. F. F. 60 años, agricultor, vecino de Potrero Grande de Buenos Aires (Región Sur-Este de Costa Rica). Buen estado nutritivo y apariencia saludable. Estuvo internado en el Hospital en diciembre de 1943, época en que se le practicó una biopsia con la cual se estableció el diagnóstico de "Blastomicosis". Habiendo tenido oportunidad de ver la preparación microscópica y la descripción que del caso hiciera Peña Chavarría en la hoja clínica perteneciente a este enfermo, lo hicimos venir con el objeto de confirmar el diagnóstico que nos parecía más probable, y que era el de Enfermedad de Lobo.

Ingresa el enfermo al Hospital el 7 de marzo de 1949 y refiere que hace más o menos 30 años notó un pequeño nódulo sobre el maleolo externo del pie izquierdo, que fué extendiéndose en superficie. Posteriormente aparecieron otros, tornándose algunos confluentes. No le han ocasionado dolor ni ardor y solamente sangran a causa de traumatismos durante su trabajo.

El enfermo presenta una placa con numerosos nódulos subepidérmicos que van del maleolo interno al externo, sobre la cara posterior del miembro inferior izquierdo; a lo largo del borde tibial hay una cadena de nódulos de diversos tamaños. En su mayoría los nódulos son consistentes, cubiertos de piel delgada, atrófica, rosada, sin vellos; no adhieren a los planos profundos, excepto en la placa maleolar; otros presentan apenas modificada la piel que los recubre.

Al nivel de la rodilla hay 3 nódulos subcutáneos, recubiertos de piel normal 
y no adheridos ni a ésta ni al hueso. En la cara interna de la pierna se palpa también una cadena de 5 nodulitos pequeños, semejantes a los anteriures (Pl. III, Figs. 1-2-3).

En resumen el aspecto clínico de esta blastomicosis encuadra perfectamente en las descripciones hechas por Lobo ( 5 y 6), FialHo (3) y da FonseCa (4).

En 1943 no presentaba adenopatía crural ni inguinal, según las observa ciones de Peña Chavarría, pero en 1949 se observa que hay un ganglio crural no doloroso del tamaño de un huevo de paloma. En el muslo ro se nota ningún nódulo, siendo las lesiones más altas, las descritas al nivel de la rodilla.

En el material obtenido por escarificación de las lesiones y examinado al microscopio, a fresco entre porta y cubre-objetos, encontramos enorme cancidad de parásitos que se presentan como células redondas con membranas refrigentes, de doble contorno y que, en contraposición a lo que comúnmente se observa con relación al Paracoccidioides brasiliensis y al Blastomyces dermatitidis, tienen dimensiones bastante uniformes (entre 7 y 14 micras) como puede apreciarse en la fotomicrografía (PI. IV, Fig. 1). Algunos parásitos revisten la forma "en cúpula" (Pl. IV, Figs. 2 y 5). Se observan escasos elementos en gemación (Pl. IV, Fig. 3), y esporádicamente cadenas de 2 a 4 células (Pl IV, Figs. 4, 5 y 6); la formación de la yema está acompañada por la membra ạta (P1. IV, Figs. 3 y 4 ) sin que en ésta puedan apreciarse los agujeros que se observan en el $P$. brasiliensis. En fragmentos de tejido retirados por biopsia $y$ colocados en $\mathrm{NaOH}$ al $40 \%$, notamos algunas células cuyo aspecto hace sospechar una gemación simultánea en 2 ó 3 partes de la membrana (Pl. IV, Figs. 7, 8 y 9). Por los caracteres anteriormente expuestos, clasificamos este hongo como Glenosporella loboi Fonseca et Leao, 1940.

No obstante haber sembrado más de 200 tubos con diversos medios de cul. tivo sólidos y líquidos, a algunos de los cuales se agregó penicilina y a otro; tiamina, usando como "inoculum" fragmentos de tejido de uno a dos $\mathrm{mm}$ de diámetro, e incubando a $37^{\circ} \mathrm{C}, 27^{\circ} \mathrm{C}$ y a temperatura ambiente, nos ha sido imposible hasta el momento obtener el cultivo del hongo en cuestión, aun cuando los tubos ya tienen más de un año de observación.

Fracasamos igualmente en las tentativas de reproducir granılomas por inoculación intratesticular en cobaya, no obstante que, siguiendo el consejo de los Dres. Cury y Goto del Lab. de Micología del Instituto Oswaldo Cruz, practicamos hasta cinco inoculaciones a un mismo animal, con intervalos de una semana.

En lo que se refiere al aspecto histopatológico de las lesiones, nuestro material fué estudiado por el personal de la Sección de Anatomía Patológica del Instituto Oswaldo Cruz y de sus detallados reportes se puede deducir la semejanza con los otros casos de Blastomicosis Queloideana anteriormente descritos, en todos los cuales llama la atención la ausencia de supuración (Pl. V, Figs. 1 a 7 ) .1

1 Queremos dejar aquí constancia de nuestro profundo agradecimiento a los Dres. C. B. Magarinos Torres, Eitel Duarte, Rita A. Almeida Cardoso y Jorge P. Guimaraes del Laboratorio de Anatomía Patológica, así como a los Dres. Area Leao, Cury y Goto del Lab. de Micología por la colaboración y estímulo que prestaron a este estudio, que aun cuando efectuado fuera del Instituto Oswaldo Cruz, es una modesta extensión de la obra que ahí se lleva a cabo. 
Nos parece interesante resaltar el hecho de que en algunas lesiones abundan las células gigantes (Pl. V, Fig. 4), mientras que en otras predominan los elementos histiocitarios e inflamatorios, (Pl. V, Fig. 5). Hay por fin nódulos subcutáneos que sospechamos sean ganglios linfáticos que han perdido totalmente su estructura original, substituída principaimente por tejido conjuntivo fibroso y parásitos (Pl. V, Figs. 6 y 7 ).

En los cortes del ganglio crural a que hicimos referencia anteriormente puede verse que "la arquitectura del ganglio linfático está conservada, mostrando una distención de los senos linfáticos medulares. Muchos folículos linfoides muestran centro germinativo desenvuelto. El aumento de los senos linfáticos parece ser debido a un ensanchamiento del retículo, más que a la abundancia de células". (P1. VI, Fig. 1).

En otros cortes de este ganglio encontramos además verdaderos nidos de histiocitos y algunas células gigantes y parásitos, localizados de preferencia en la periferia del ganglio (Pl. VI, Figs. 1 a 6 ). Queda pues demostrada la diseminación por vía linfática en la Blastomicosis Queloideana, cuando menos en nuestro caso, cuya evolución data de más de 30 años. ${ }^{1}$

\section{RESUMEN}

Después de dar las referencias de los casos de Blastomicosis anteriormente descritos en este país, se estudia un nuevo caso (el tercero) de Enfermedad de Lutz, que se clasificó como perteneciente a la "forma mixta" de Aguiar Pupo y no obstante la terapéutica empleada tuvo éxito letal.

Con respecto al Paracoccidioides brasiliensis se discute la posibilidad de que el mismo dé endosporulación, comprobándose que vacuolas de substancias lipoides puecien, en determinadas condiciones, semejar este proceso de reproducción.

Se esíudia además un caso de Enfermedad de Lobo; el primero que se registra en Costa Rica y fuera de la región Amazónica, comprobándose que esta blastomicosis puede diseminarse, al principio, por los linfáticos superficiales y después de una larga evolución, por los linfáticos profundos, pudiendo interesar inclusive los ganglios de la región crural.

\section{RESUME}

Apres avoir fait mention des cas de Blastomycoses qu'on été decrits anterieurement dans ce pays, on presente un nouveau cas (le troisieme) de Maladie de Lutz, qu'on a consideré comme une "Forme Mixte" de la classification de Aguiar Pupo et qui mourut malgré la thérapeutique employée.

I En el caso de Guimaraes y Macedo a que hicimos referencia anteriormente, y en el cual se cita el nuestro, se pudo confirmar también la diseminación linfática del parásito. 
Quant au Paracoccidioides brasiliensis on discute la possibilité qu'il puisse se reproduire par endosporulation car on a demontré que des vacuoles remplies des sustances lipoides peuvent donner, dans certaines conditions l'impression de ce procés de reproduction.

On étudie au meme temps, un cas de Maladie de Lobo, le premier qui a été registré a Costa Rica et le premier aussi, qu'on a trouvé en dehors de la region de l'Amazone. On demontre que cette blastomycose pert se propager, au debut, par les lymphatiques superfitiels, et qu'apres une longue evolution, la dissemination peut etre faite par les lymphatiques profonds, jusquaux ganglions de la region crurale.

\section{SUMMARY}

After making reference to the cases of blastomycosis previously described in this country, a new case (third) of Lutz' disease was studies and classified as belonging to the "forma mixta" of Aguiar Pupo's. In spite of the therapeutics used the patient died.

With regard to the $P$. brasiliensis, the possibility was considered of its effecting endosporulation, and it was verified that vacuoles of fatty substances may, under certain conditions, resemble this process of reproduction.

A case of Lobo's disease was also studied, the first one recorded in Costa Rica and outside the Amazonic area, stablishing the definite possibility that this blastomycosis is first distributed through the superficial lymphatic vessels and. after a long evolution, through the deep lymphatic vessels.

\section{ZUSAMMENFASSUNG}

Nachdem die früher in diesem Lande (Costa Rica) beschriebenen Fälle von Blastomycose erwähnt wurden, prüft man einen neuen Fall (den dritten) von Lutzscher Erkrankung, den man als zur "gemischten Form" von Aguiar Pupo gehörig klassifiziert. Trotz der angewandten Therapie ist der Ausgang letal.

Bezüglich der Paracoccidioides brasiliensis erwähnt man Möglichkeit, dars dieser Endosporulation zeige. Bewiesen wird, dass Vakuolen von lipoiden Substanzen unter bestimmten Bedingungen diese Art von Reproduktion vortäuschen können.

Man prüft ferner einen Fall von Loso-erkrankung, der erste, den man in Costa Rica und ausserhalb der Amazonenregion verzeichnet. Es wird nachgewiesen, dass diese Blastomycose sich anfangs über die oberflächlich gelegenen Lymphwege ausbreiten kann und später nach langer Entwicklung über die tiefer liegenden Lymphwege 


\section{REFERENCIAS BIBLIOGRAFICAS}

1. Aguiar Pupo. Cit. en Almeida, (2).

2. AlMEIDA, F. P.

1939. Mycologia Médica XII + 709 pp. Companhia Melhoramentos de Sao Paulo, Sao Paulo.

3. Fialmo, $\mathrm{A}$.

1938. Blastomicose do tipo "Jorge Lobo". O Hospital 14 (4): $903-918$.

4. FonseCA, O. DA

1943. Parasitologia Médica. Tomo I. 737 pp. Editora Guanabara. Rio de Janeiro.

5. Lово, J.

1933. Contribuicao ao estudo das blastomicoses. An. Brasil. Dernat. e Sifilograf. 8:43-57.

6. Lово, J.

1949. Blastomicoses. Estudo especial da "Doenca de Jorge Lobo". Arq. Med. Cir. Pbco. 1 (1): 3-35.

7. Peña Chavarría, A. \& Rotter, W.

1932. Observaciones sobre la cromoblastomicosis y la blastomicosis en Costa Rica. 18 pp. XII Conferencia Med. Imprenta Gutenberg. San José.

8. Peña Chavarría, A. \& Rotter, W.

1934. Consideraciones anatomopatológicas y clínicas de la blastomicosis en Costa Rica. Separata de Rev. Med. Latino-Americana, 19 (226): 21 pp.

9. Peña Chavarría, A. \& Ovares, J. C.

1941. Un caso de blastomicosis cutánea producicia por Torulopsis mitis. Rev. Med. Costa Rica. 4 (35): 475-489.

10. Peña-Chavarría, A., Agullar, M., Fallas, M. \& Castro, A.

1949. Apuntes sobre un nuevo caso de granuloma paracoccidioides en Costa Rica. Rev. Med. Costa Rica 8 (179): 369-375.

11. ROTTER, W.

1934. Micosis en Costa Rica. Memorias del Segrnndo Congreso Médico Centroamericano reunido en San José de Costa Rica. Pág. 196-203. Imprenta Nacional. San José.

12. Rotter, W. \& Peña-Chavarría, A.

1933. Untersuchungen über Blastomykosen in Costa Rica, Arch. Scbifs-Trop. - Hyg. $37: 1-10$.

13. Rotrer, W. \& Peña-Chavarría, A.

1934. Weitere Untersuchungen über Blastomykosen in Costa Rica. Arch ScbiffsTrop. - Hyg. $38: 406-417$. 


\section{PLANCHA I}

\section{ENFERMEDAD DE LUTZ}

Fig. 1: R. Z. Aspecto del paciente en diciembre de 1948. Foto Claudio Jiménez.

Fig. 2: Paracoccidioides brasiliensis. Cultivo de ocho semanas en Sabouraud maltosado de la cepa aislada de R. Z.

Fig. 3: Corte de intestino de R. Z. mostrando gran cantidad de criptosporas. Col. Hematoxilina-Eosina, $450 \mathrm{X}$.

Fig. 4: P. brasiliensis. Pus de la lesión de la mejilla derecha. A fresco, $980 \mathrm{X}$.

Fig. 5: Histiocito con varios parásitos fagocitados, raspado de la úlcera del surco nasogeniano. Col. Giemsa, $980 \mathrm{X}$.

Figs. 6,

7 y 8: Células con 2, 3 y 4 núcleos respectivamente, y parásitos en gemación, en el interior de sus citoplasmas. Se observa la cromatina de los parásitos localizada en masas que ocupan diversas posiciones. El mismo ma* terial de la microfoto anterior. Giemsa, $980 \mathrm{X}$. 


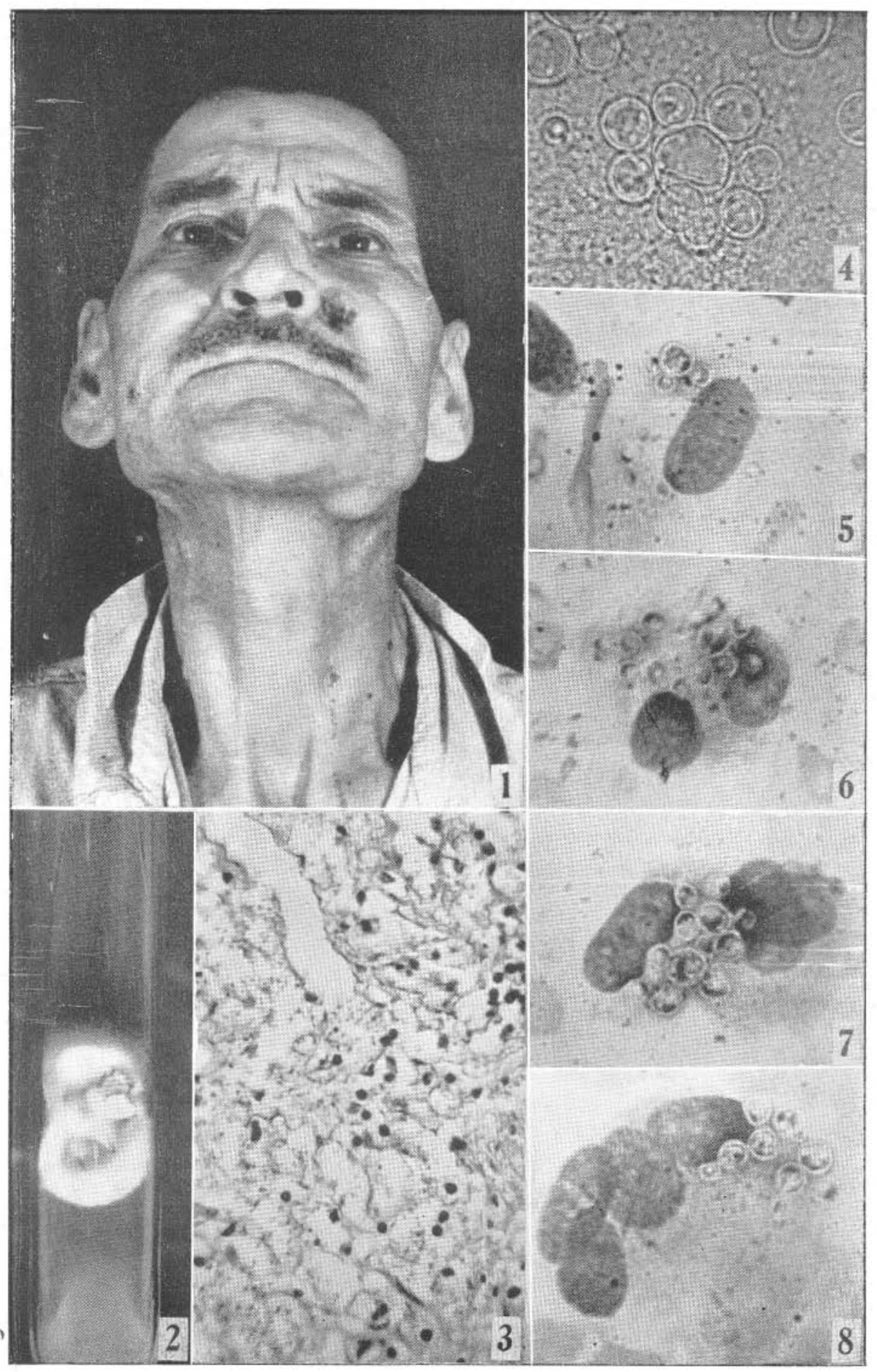


PLANCHA II

\section{FNFERMEDAD DE LUTZ}

Fig. 1: Corte de encía de R. Z en el que se ve una célula de $P$. brasiliensis en criptosporulación. Col. Henuatoxilina férrica, $980 \times$. (Foco en el ecuador de la célula).

Fig. 2: La célula de la foto anterior focalizando el hemisferio superior de la misma, en el que pueden apreciarse los pequeños agujeros en la membrana, a través de los cuales salen las criptosporas.

Fig. 3: P. brasiliensis. Célula $\in \mathrm{n}$ criptosporulación. Raspado de una lesión cutánea. Col. Giemsa, 980 X:

Fig. 4: Célula con aspecto semejante a endosporulación. Raspado de la lesión de la encía. A fresco, $980 \mathrm{X}$.

Fig. 5: La misma microfoto anterior ampliada 3,5 $\mathrm{X}$.

Fig. 6: Célula con dos yemas y un tubo germinativo. Pus del ganglio submaxilar, conservado 6 días a temperatura ambiente. Se aprecian las vacuolas de grasa en el interior de la célula y en el tubo germinativo. Las yemas muestran una única vacuola cada una. A fresco, $980 \mathrm{X}$.

Fig. 7: Otro aspecto en el mismo material de la microfoto anterior. A fresco, $980 \mathrm{X}$. 


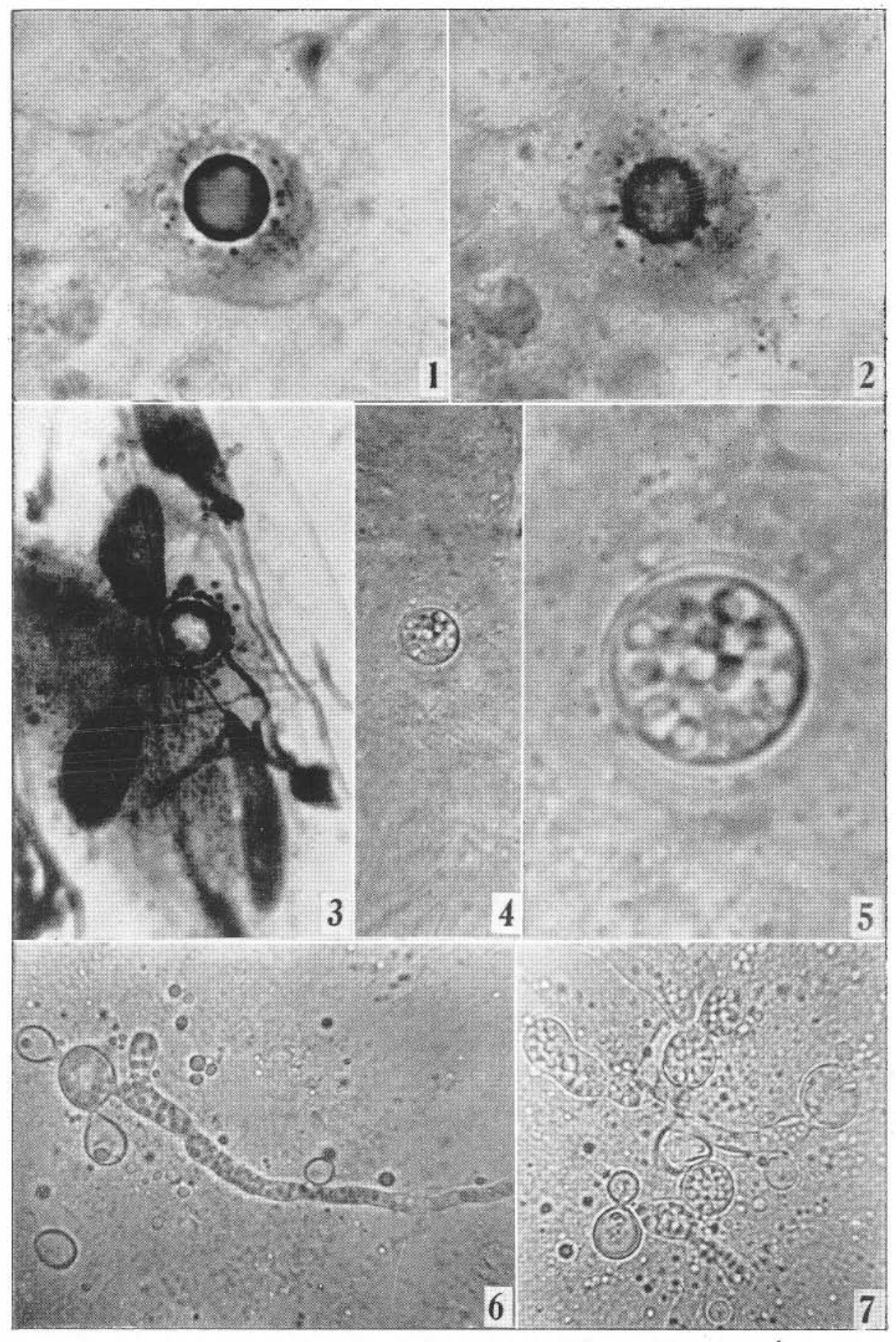


FLANCHA III

\section{ENFERMEDAD DE LOBO}

Figg. 1-2 y 3: A. F. F. Vistas externd, frontal e interna de la pierna izquicrda. Sc aprecian lis vegetacionos de aspecto queloideano de varios tamaños. In la ligig. 1 pueden verse al ģunos nódulos subcutáncos a la altura de la rodilla, cubiertos por piel normal, y en la lesión que cubre ol malcolo externo, lat cicatriz en el sitio donde fué practicalda una biopsia en 1943 . 


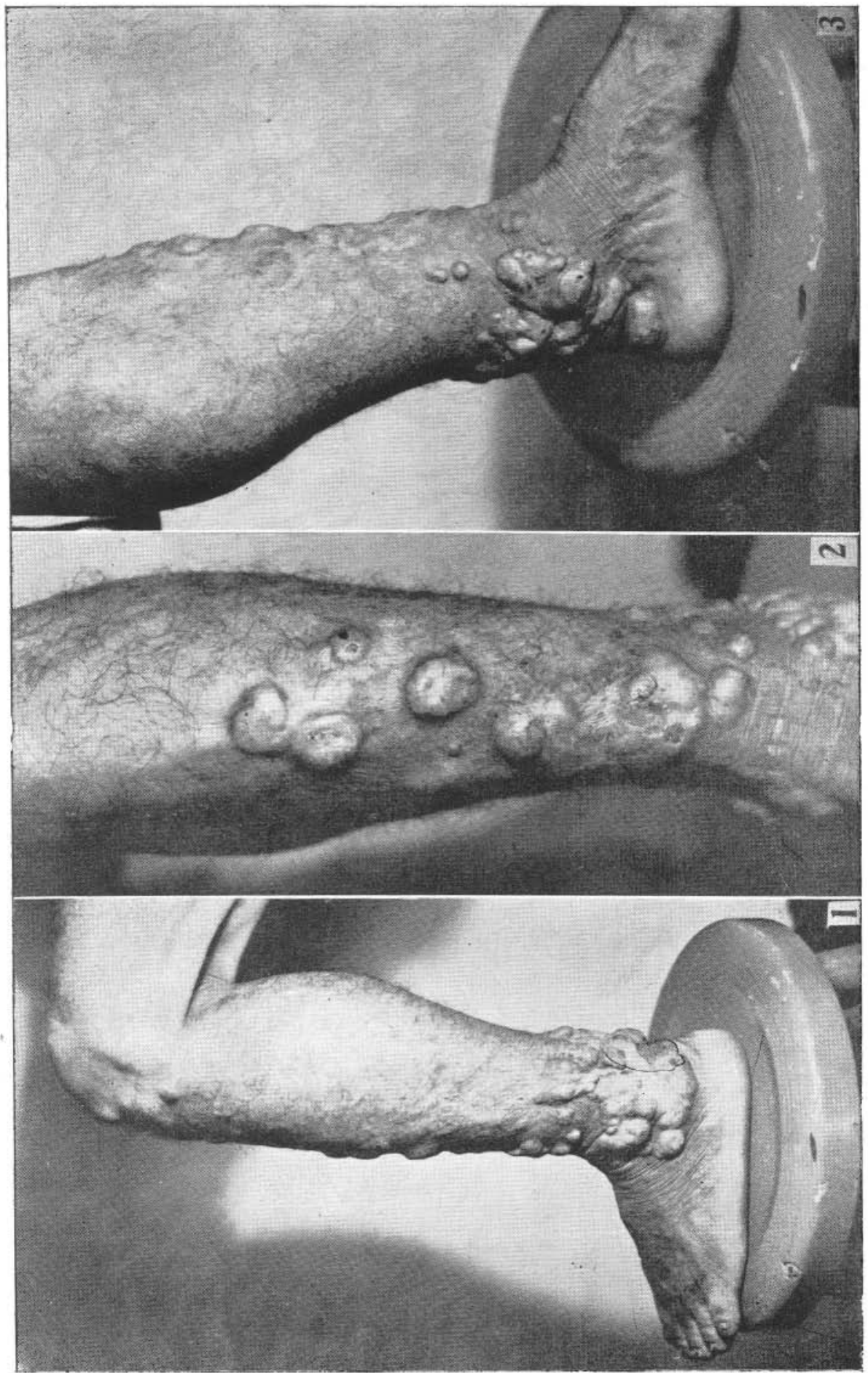




\section{PLANCHA IV}

\section{ENFERMEDAD DE LOBO}

Fig. 1: Glenosporella loboi. Raspado de un nódulo de la cara. anterior de la pierna de A. F. F. Obsérvese la abundancia del parásito y la uniformidad de sus dimensiones. A fresco, 450 $\mathrm{X}$.

Fig. 2: Raspado de lesión cutánea. Una forma redonda y una forma "en cúpula". A fresc $\bullet 980 \mathrm{X}$

Fig. 3: Raspado de lesión cutánea. Célula en gemación. Puede apreciarse que la membrana acompaña la yema. A fresco, $980 \mathrm{X}$.

Fig. 4: Raspado de lesión cutánea. Cadena de 3 elementos el último de los cuales está iniciando la formación de una yema. A fresco, $980 \mathrm{X}$.

Fig. 5: Nódulo subcutáneo de la región rotuliana en $\mathrm{N} 3 \mathrm{OH}$ al $40 \%$. Cadena de 3 elementos uno de íos cuales tiene forma en cúpula. $980 \mathrm{X}$.

Fig. 6: Mismo material de la microfoto anterior. Cadena ramificada de 5 elementos. $\mathrm{NaOH}$ al 40 \%, 980 $\mathrm{X}$

Fig. 7: Mismo material. Célula con aspecto de gemación bipolar. $\mathrm{NaOH}$ al $40 \%$. $980 \mathrm{X}$.

Fig. 8: Mismo material. Célula con dos yemas vecinas. $\mathrm{NaOH}$ $40 \%, 980 \mathrm{X}$.

Fig 9: Mismo material. Célula que aparentemente di gemación en 3 puntos diversos de su membrana. 


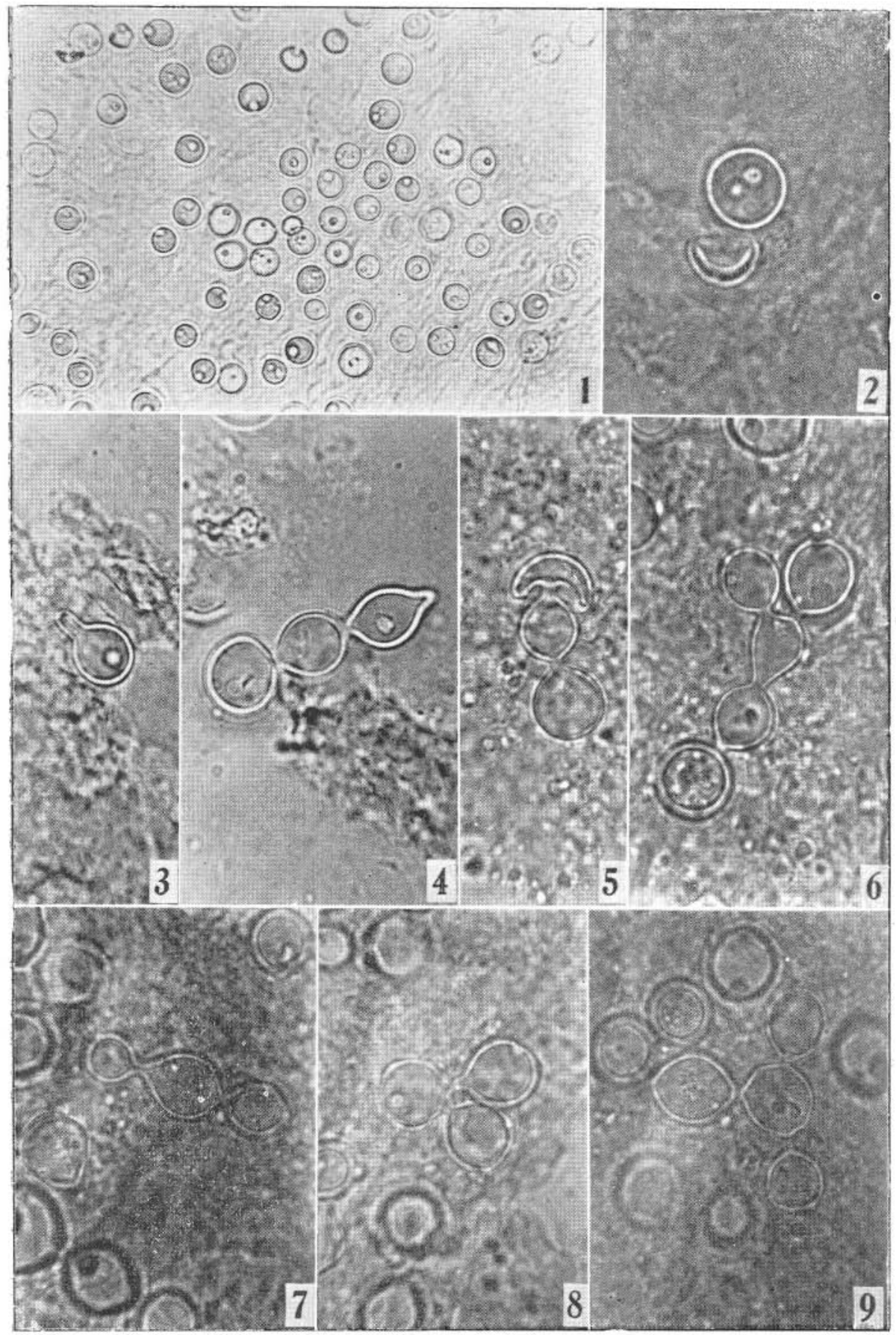




\section{PLANCHA V}

\section{ENFERMEDAD DE LOBO}

Fig. 1: A. F. F. Corte de un nódulo cutáneo con la piel muy adelgazada. Se observa gran cantidad de parásitos la mayoría de los cuales no toman los colorantes. En la epidermis se observa una célula gigante rodeada de parásitos y células epitelioides, y en otro punto un grupo de parásitos en la vecindad de la capa córnea. Hematoxilina-eosina, $70 \mathrm{X}$.

Fig. 2: Detalle de la microfoto anterior. Se aprecia el conjunto de parásitos cerca de la capa córnea. Hematoxilina-eosina, $315 \mathrm{X}$.

Fig. 3: Detalle de la microfoto de la Fig. 1 que muestra el "microgranuloma" intraepidérmico. Hematoxilina-eosina, $315 \mathrm{X}$.

Fig. 4: Otro aspecto del corte de la Fig. 1. Región en que predominan las células epitelioides $e$ inflamatorias. Hematoxilina-eosina, $315 \mathrm{X}$.

Fig. 5: Otro aspecto del corte de la Fig. 1. Región en la cual hay gran cantidad de células gigantes. Hematoxilinaeosina. $315 \mathrm{X}$.

Fis. 6: Corte de un nódulo subcutáneo de la región rotuliana, cubierto por piel normal. Se aprecia su estructura fibrosa y la enorme cantidad de parásitos. Hematoxilina-eosina, $70 \mathrm{X}$.

Fig. 7: Detalle del mismo corte anterior. $315 \mathrm{X}$. 


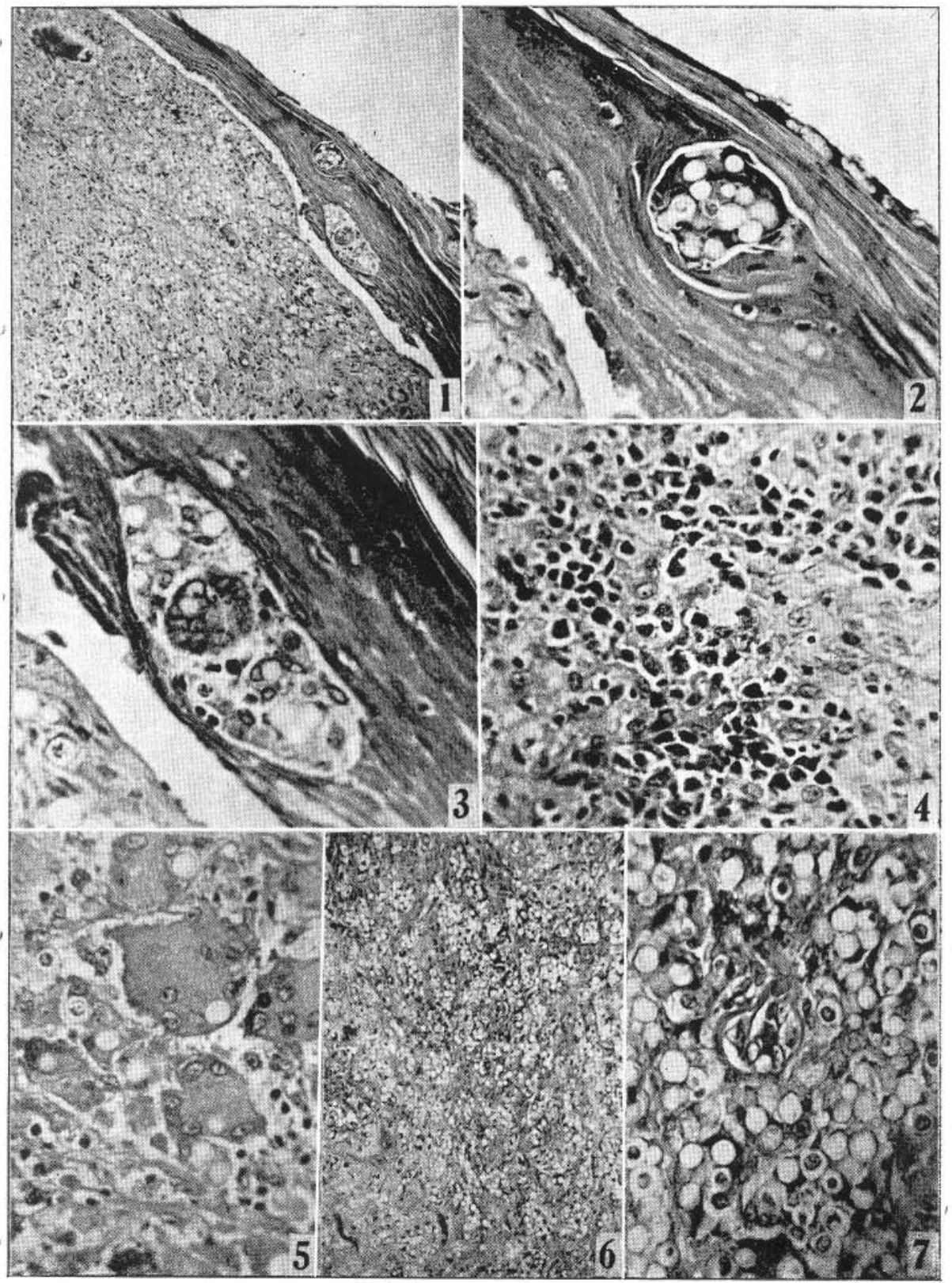



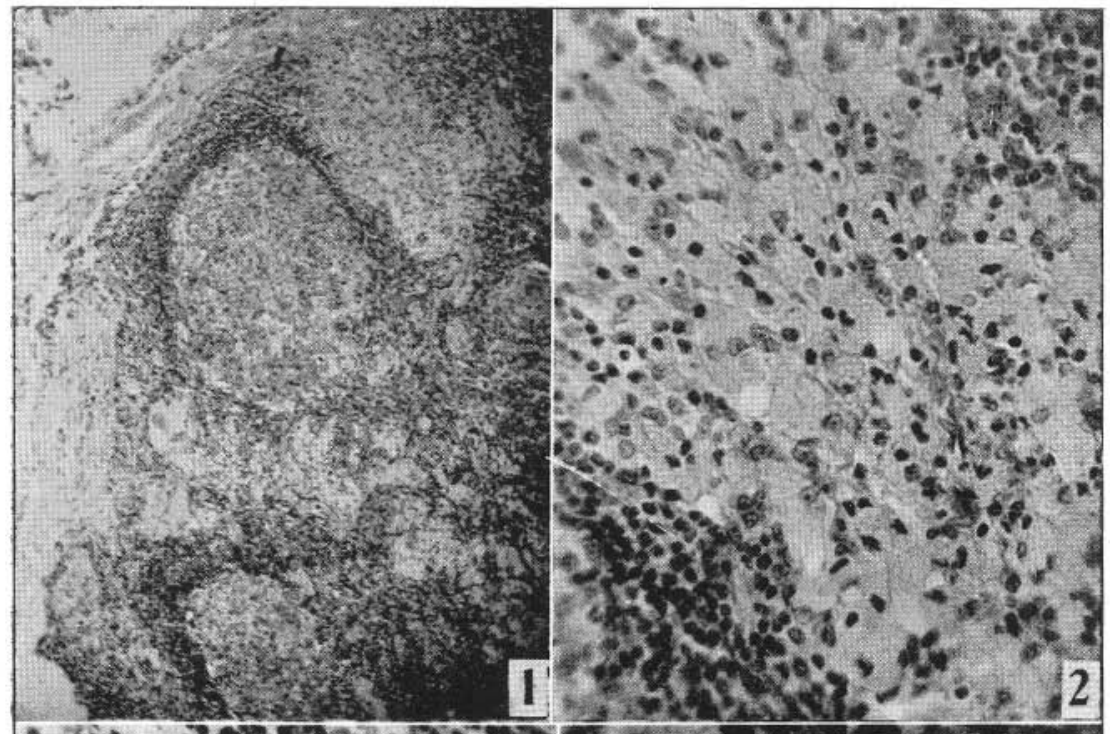

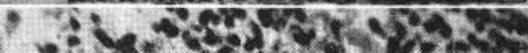

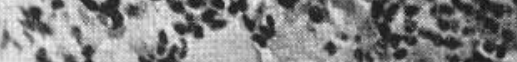

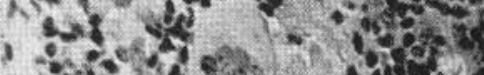

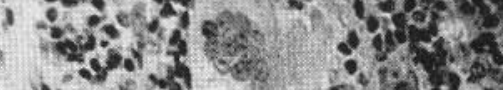
- is

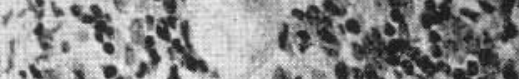

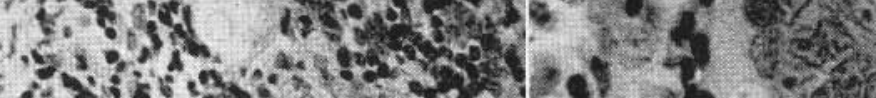

ins

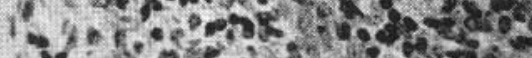
It:

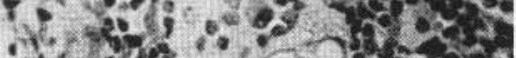

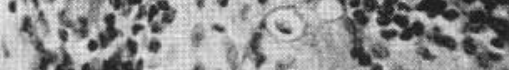

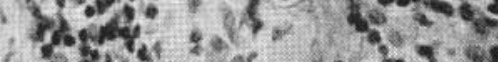

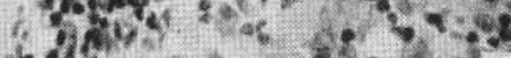

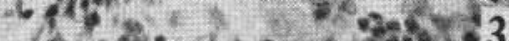

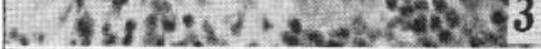

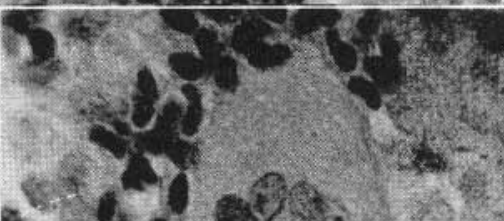

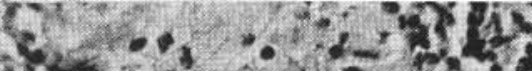

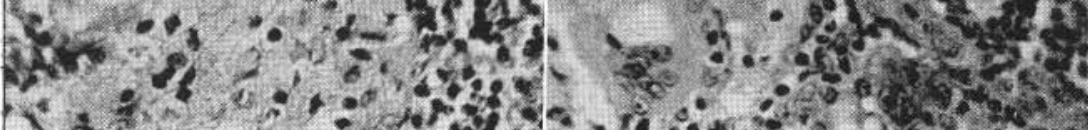

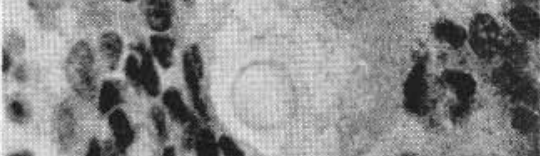
\%, is 1 ins.

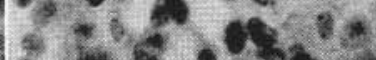

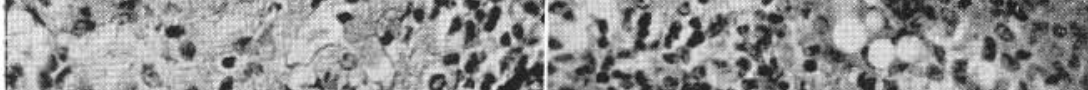

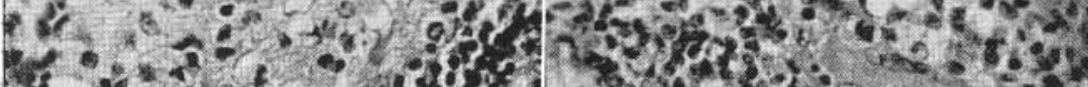

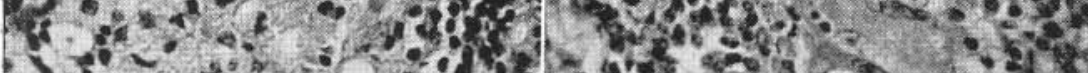

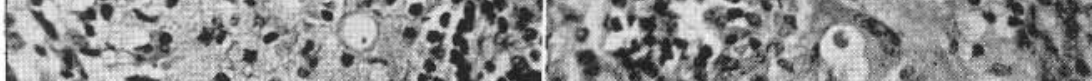

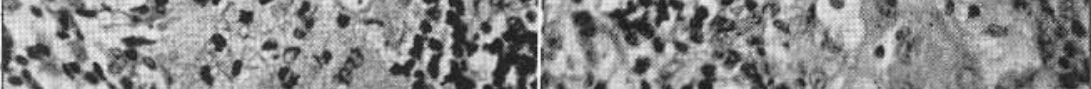

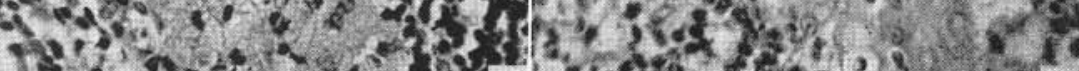

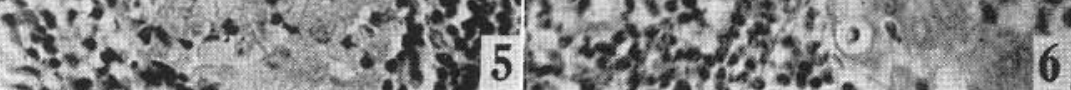

